\title{
Censo Demográfico e Paradados: \\ Em Busca da Melhor Experiência para o Usuário
}

\section{Demographic Census and Paradata: Searching for the Best User Experience}

\author{
Patricia Zamprogno Tavares, Universidade Federal do Estado do Rio de Janeiro \\ (UniRio) e Instituto Brasileiro de Geografia e Estatística (IBGE) \\ pztavares@gmail.com
}

\author{
Luiz Agner, Instituto Brasileiro de Geografia e Estatística (IBGE); Faculdades \\ Integradas Helio Alonso (Facha) \\ luizagner@gmail.com
}

\author{
Simone Bacellar Leal Ferreira, Universidade Federal do Estado do Rio de Janeiro \\ (UniRio) \\ simone@ uniriotec.br
}

\section{Resumo}

Este texto tem como objetivo apresentar resultados preliminares de pesquisa de doutorado, desenvolvida com a proposta de avaliar a experiência do usuário (UX) durante o preenchimento de questionários na web, aplicados a censos demográficos. Questionários via internet para pesquisas censitárias devem apresentar avançados atributos de usabilidade de modo a facilitar o seu preenchimento pelos cidadãos brasileiros. Neste contexto, pretende-se avaliar se os paradados - registros de interações entre os usuários e o próprio questionário - podem apontar inconsistências nos instrumentos de coleta dos censos. Em oito bases online, foram realizadas buscas para o mapeamento de estudos científicos realizados sobre o emprego de paradados. Os resultados trouxeram importantes insights. Concluiu-se que os paradados podem atuar como uma importante ferramenta complementar na avaliação da experiência do usuário em pesquisas censitárias online.

Palavras-chave: Paradados, Usabilidade, Experiência do Usuário, UX, Censo Demográfico.

\begin{abstract}
This paper aims to present preliminary results of doctoral research, developed with the proposal of evaluating the user experience $(U X)$ during the completion of web-based questionnaires applied to demographic censuses. Web-based questionnaires for census research should present advanced usability attributes in order to facilitate their completion by Brazilian citizens. In this context, we intend to evaluate whether the paradata - records of interactions between users and the questionnaire itself - can point to inconsistencies in the census collection instruments. Eight online databases were searched in order to map scientific studies conducted on the use of paradata. The results brought important insights. We concluded that paradata can act as an important complementary tool in the evaluation of user experience in online census surveys.
\end{abstract}

Keywords: Paradata, Usability, User Experience, UX, Demographic Census. 


\section{Introdução}

Com realização periódica de coleta de dados, o censo demográfico brasileiro é uma das principais fontes de referência em nosso País para a produção de informações sobre o perfil e as condições de vida da população. É o estudo estatístico realizado, em bases decenais, na maioria dos países, que monitora mudanças e características socioeconômicas das suas populações, vitais para o entendimento da economia e da sociedade (UNECE, 2015).

Os censos demográficos são realizados pelos órgãos oficiais de produção estatística e, devido à sua complexidade e dimensão, exigem grande esforço e investimento para sua realização. $\mathrm{O}$ método tradicional de coleta de dados populacionais, caracterizado por visitas presenciais a domicílios, realizadas por recenseadores (entrevistadores contratados temporariamente somente para este fim), vem sendo cada vez mais questionado. Traduzindo em números, em 2019, o censo demográfico brasileiro foi orçado, pelo Instituto Brasileiro de Geografia e Estatística (IBGE), em R \$ 3,4 bilhões, para uma população estimada em 212,2 milhões de pessoas. A estrutura total da operação pode envolver mais de 250 mil profissionais com o objetivo de visitar aproximadamente 70 milhões de endereços, nos 5.570 municípios do território nacional (IBGE, 2019).

As maiores dificuldades dos censos demográficos relacionam-se ao custo e à complexidade, que representam desafios para qualquer país. A fase de coleta de dados poderá custar até $75 \%$ do orçamento da operação censitária, com o recrutamento de inúmeros recenseadores e o seu deslocamento para realizar centenas de milhares ou mesmo milhões de entrevistas em áreas dispersas geograficamente (PASC, 2014).

Outro fato desfavorável envolve a disposição da população em participar das pesquisas, que vem caindo a cada ciclo censitário. Muitos cidadãos ficam com receio de abrir a porta para os recenseadores, o que torna mais difícil realizar este tipo de coleta de dados (NORDHOLT, 2018).

Por fim, a crise da Covid-19 reforçou a necessidade de repensar as coletas presenciais, e obrigou os institutos de estatística a adiar ou até considerar o cancelamento de seus censos tradicionais (CCSA, 2020; IBGE, 2020).

Essas são dificuldades que vêm levando os países a estudar outras formas de coletas eletrônicas, sem o auxílio de recenseadores (IBGE, 2020; UNSD, 2020). Uma das alternativas de coleta eletrônica sem recenseadores pode ser a adoção de formulários online, o que vem sendo bem considerado por comitês estatísticos internacionais (UNECE, 2015). Os institutos nacionais de estatística vêm avançando na migração para a Internet, e vêm estimulando a população a responder aos questionários dos censos na Web (PASC, 2014). No Brasil, o IBGE está investindo na internet como forma de coleta para o censo demográfico: o fato representa uma inovação no modo de se obter dados junto aos cidadãos brasileiros, pois, em nosso País, a operação censitária sempre foi acompanhada por recenseadores (IBGE, 2018).

Assim, a Web (como meio de coleta de dados para o censo) acarretará uma profunda mudança no modelo de preenchimento. Sem a figura do recenseador, treinado para ser capaz de dar suporte, os cidadãos precisarão compreender e interagir de modo eficaz com o questionário, por conta própria. Os impactos da nova modalidade devem ser mensurados e as atividades conduzidas com 
cautela, para atender aos requisitos dos cidadãos no preenchimento dos questionários eletrônicos, de modo a garantir a qualidade dos dados (UNECE, 2015).

Dificuldades ou limitações no uso da Web são importantes preocupações. Além disso, problemas técnicos podem acarretar aumento do abandono de questionários nas pesquisas online. Portanto, a comunicação entre cidadão e questionário eletrônico precisa ser transparente e intuitiva, partindo de uma adequada experiência. Estudos de usabilidade e UX (experiência do usuário) são ainda mais recomendáveis nas entrevistas autoadministradas, visando a aumentar o grau de satisfação dos cidadãos no preenchimento dos questionários e a aprimorar a sua experiência (KACZMIREK, 2008).

No contexto da qualidade em pesquisas, os institutos de estatística vêm, gradualmente, implementando a captura de dados auxiliares - os paradados - para monitorar as coletas de dados. Paradados são registros de movimentos e ações realizadas (como teclas digitadas, acionamento de botões ou cliques de mouse) durante o preenchimento de questionários e podem servir como subsídios para compreendermos o processo de coleta de dados e estudar o comportamento do usuário (DURRANT \& KREUTER, 2013; COUPER, 2017).

Diante do cenário exposto, o objetivo deste trabalho é iniciar com o mapeamento da pesquisa atual sobre paradados e mapear a sua aplicação no aprimoramento da experiência do usuário ao preencher pesquisas censitárias. Como o Brasil vem realizando testes regulares para a migração do censo tradicional para a abordagem online (IBGE, 2018), o intuito desta pesquisa é apoiar com conhecimento científico a construção de um questionário online com adequada usabilidade para oferecer à população uma opção amigável, compreensível e eficiente.

\section{Métodos de Coleta de Dados em Censos Demográficos}

O instrumento típico de coleta dos dados em censos demográficos é o questionário. Na coleta dos dados por questionários (em papel ou eletrônicos), as perguntas podem ser respondidas de diversas maneiras: através de entrevistas pessoais ou por telefone, mediados por recenseadores ou podem ser respondidos diretamente pelo cidadão, através dos questionários recebidos pelos correios ou respondidos pela Internet (NORDHOLT, 2018). Para acompanhar o foco do presente artigo, será descrito o método de coleta apoiado por questionários na Web.

No método CAWI (Computer-Assisted Web Interviewing, ou Autoentrevista Assistida pela Web), a coleta de dados é realizada sem a intervenção do recenseador: o próprio cidadão lê e responde às perguntas que surgem na tela, em questionários disponíveis na Internet.

A adoção da Internet como um modelo mais eficiente de coleta de dados vem sendo discutida no mundo inteiro como forma de facilitar a resposta aos censos, além de oferecer uma imagem mais moderna aos institutos de estatística. Por outro lado, mesmo levando em consideração experiências positivas, a taxa de respostas online é variável entre os países e nem todos tiveram boas taxas em experiências na Internet (INE, 2014).

Considera-se estimular as respostas online, mas não é possível prever a aceitação da população. Isto depende de características particulares de cada população, como a proporção de 
pessoas que possuem acesso à Internet e como a sociedade se comporta perante tecnologias modernas. A abordagem online corre o risco de excluir pessoas e famílias, com a predominância de certos públicos que preferem preencher os formulários via Internet, como os mais jovens que residem em áreas urbanas (INE, 2014; UNECE, 2015).

\section{A importância da usabilidade e UX para o método CAWI}

A usabilidade é um requisito de qualidade dos softwares que visa tornar transparente e intuitiva a comunicação entre o usuário e o sistema. Define diretrizes que determinam se o manuseio de um produto é fácil e rapidamente aprendido, dificilmente esquecido, não provoca erros operacionais, oferece alto grau de satisfação e eficientemente resolve tarefas para as quais ele foi projetado (LEAL FERREIRA \& NUNES, 2008). Além da usabilidade, outros critérios podem ser adicionados à proposta da experiência do usuário, como: emoção, credibilidade, estética, performance e segurança. A UX pode incentivar os usuários a utilizarem o produto (ou serviço) não só porque têm facilidade para atingir seus objetivos, mas porque estes transmitem emoções positivas (AGNER, 2018).

A aplicação de princípios de usabilidade deve ser prioritária na migração de questionários em papel para questionários eletrônicos: o processo requer muito mais do que simplesmente converter o instrumento de papel em um formato digital (KACZMIREK, 2008). O design do questionário eletrônico, desde a apresentação visual das perguntas e respostas até as suas funções de suporte e ajuda devem ser concebidos cuidadosamente (TAVARES, 2011). É essencial que seu desenvolvimento seja centrado nas necessidades do usuário - no caso, os cidadãos - visto que a interpretação das perguntas e das opções de resposta pode estar associada ao design visual do questionário na tela.

Cabe ressaltar que os recenseadores recebem treinamento para operar e interpretar o questionário, mas, no caso dos questionários preenchidos pelos cidadãos, não há treinamento. Portanto, estudos de usabilidade são ainda mais recomendáveis para questionários auto administrados, ou seja, onde o cidadão o responde por conta própria, sem o suporte direto de um recenseador (KACZMIREK, 2008).

Sendo assim, se os erros de usabilidade não forem tratados desde a concepção do questionário, a inovação tecnológica poderá gerar efeitos reversos na qualidade dos dados e na imagem do próprio censo. É fundamental criar uma interface com navegação simples, onde erros de entrada de dados possam ser minimizados, com textos claros e com instruções de ajuda úteis, que sejam fáceis de usar e não dependam do conhecimento prévio de termos técnicos. Ao reduzir a probabilidade de erros, de interpretações imprecisas e ao evitar problemas na interação, melhorase a qualidade da própria pesquisa (KACZMIREK, 2008) e contribui-se para o grau de satisfação dos usuários no preenchimento dos questionários. 


\section{Paradados: dados auxiliares para monitorar a experiência do usuário}

Os institutos de pesquisas estatísticas vêm investindo na capacitação e exploração dos paradados a fim de monitorar e gerenciar suas coletas de dados em larga escala. O termo foi introduzido pela primeira vez em 1998, pelo pesquisador Mick Couper, da Universidade de Michigan. Couper propôs o termo paradata para referenciar dados auxiliares, capturados durante a coleta de dados, que descrevem e ajudam a entender o processo eletrônico e os movimentos e ações do usuário realizados nos questionários (DURRANT \& KREUTER, 2013; COUPER, 2017).

Um tipo comum de paradados é o tempo de resposta para concluir cada pergunta ou a entrevista completa, mas também podem ser registradas sequências de alterações de respostas, capturas de pressionamento de teclas, navegação pelo questionário, entre outros. Pode-se ainda analisar a quantidade de vezes que janelas de erro ou ajudas foram exibidas, ou identificar o modelo do dispositivo que está sendo utilizado.

Couper ressalta a importância e os benefícios dos paradados, que oferecem riqueza de informações sobre a experiência do usuário durante o processo de preenchimento do questionário. Entender esse comportamento e empregar paradados, de maneira sistemática, para avaliar o desempenho do usuário, podem ajudar a identificar melhorias necessárias nas aplicações de coleta de dados (COUPER, 2017).

\section{Método empregado nesta pesquisa}

Com o objetivo de conhecer o estado da arte sobre o tema "paradados" e seu emprego para o aprimoramento da experiência do usuário durante o preenchimento de questionários online, foi realizado o mapeamento sistemático da literatura, a fim de buscar, através de uma metodologia confiável e rigorosa, uma avaliação geral de estudos disponíveis e relevantes sobre esta área de pesquisa.

Utilizou-se o método de Revisões Sistemáticas de Literatura em Engenharia de Software (em inglês: Systematic Literature Reviews in Software Engineering, ou SLR) - com base na proposta de Kitchenham e Charters (2007) - que propõe regras para revisões de publicações científicas. Segundo o SLR, o processo é realizado através de três etapas, que nortearam o presente mapeamento: planejamento, condução e análise dos resultados.

$\mathrm{Na}$ etapa de planejamento, definiu-se o escopo do estudo através da formulação de questões de pesquisa para o mapeamento, as palavras-chave de busca e a estratégia usada para a extração dos resultados em bases de dados eletrônicas.

A partir de uma questão primária - "Como os paradados auxiliaram os pesquisadores nas análises de qualidade de questionários online? - foram elaboradas quatro questões de pesquisa secundárias dentro do contexto da questão da pesquisa primária: 
- Quais os centros de pesquisa e os pesquisadores que mais se destacam no estudo de paradados coletados em questionários online?

- Como os estudos de paradados vêm se desenvolvendo ao longo dos anos?

- Quais são as bases acadêmicas que mais apresentam o tema?

- Quais são as contribuições desses estudos para o cidadão?

Após a definição das questões de pesquisa, foram extraídas duas palavras-chave significativas para a extração dos dados nas bases ("paradata", em inglês e "paradados", em português). Optouse por realizar a busca nas seguintes bases acadêmicas: ACM Digital Library (base que cobre questões tecnológicas, educacionais e sociais da ciência da computação); IEEE Digital Library (engloba engenharia, robótica e computação), SciELO (contém periódicos científicos da América Latina e Caribe), ScienceDirect (engenharias e ciências físicas, biológicas, saúde, sociais e humanas), Scopus (abrange campos científicos, técnicos e sociais), SpringerLink (estatística, ciências da computação e ciências sociais, entre outros) e Web of Science (contém dados de diferentes disciplinas acadêmicas). Além da área tecnológica, foi incluída a SAGE Open no mapeamento, uma base eletrônica que contempla uma coleção de jornais e periódicos voltados para as comunidades de ciências comportamentais e sociais.

$\mathrm{Na}$ etapa de condução de buscas, foram acessados conteúdos científicos das bases por intermédio do Portal de Periódicos da CAPES. Para conduzir o mapeamento e categorizar a grande quantidade de estudos de forma organizada, utilizou-se o Parsifal (PARSIFAL, 2021), uma ferramenta online que auxilia em processos de revisão sistemática. No Parsifal foram importados os arquivos BibTex gerados pelas bases, criando um banco de dados único.

A partir da leitura do título, do resumo e das palavras-chave, foi avaliado se a publicação contemplou os critérios de seleção. A Tabela 1 apresenta a triagem, com os totais de aceitos, rejeitados e duplicados no mapeamento.

\begin{tabular}{|l|l|r|r|r|r|}
\hline \multirow{2}{*}{ BASES } & \multirow{2}{*}{ Site } & \multirow{2}{*}{ Total } & \multicolumn{3}{c|}{ Avaliação } \\
\cline { 4 - 7 } & & & Aceito & Rejeitado & Duplicado \\
\hline ACM Digital Library & https://www.acm.org & 93 & 29 & 42 & 22 \\
\hline IEEE Digital Library & https://www.ieee.org & 5 & 0 & 0 & 5 \\
\hline SAGE Open & https://journals.sagepub.com & 191 & 30 & 81 & 80 \\
\hline SciELO & http://www.scielo.org & 1 & 1 & 0 & 0 \\
\hline ScienceDirect & https://www.sciencedirect.com & 82 & 9 & 65 & 8 \\
\hline Scopus & https://www.scopus.com & 264 & 81 & 162 & 21 \\
\hline Springer Link & https:///ink.springer.com & 218 & 36 & 165 & 17 \\
\hline Web of Science & https://webofknowledge.com & 52 & 1 & 6 & 45 \\
\hline \multicolumn{1}{|c|}{ TOTAL } & & 906 & 187 & 521 & 198 \\
\hline
\end{tabular}

Tabela 1 - Quantitativos de textos científicos selecionados para o mapeamento (fonte: os autores)

Conforme indica a tabela 1 , foram aceitos 187 estudos, que foram novamente analisados. Neste caso, foram catalogados somente os estudos que contemplavam paradados de questionários 
online, respondidos pelo informante (sem a ajuda de um recenseador). Após esta segunda análise, foram lidos 104 estudos completamente. Depois desta leitura, foram selecionados trinta e seis estudos pertinentes.

Análise dos resultados segundo o método Systematic Literature Reviews in Software Engineering (SLR)

A leitura dos trinta e seis artigos selecionados gerou resultados que possibilitaram responder às questões propostas no planejamento deste mapeamento. Por serem mais simples e diretas, as perguntas secundárias foram respondidas, primeiramente:

Pergunta 1-Quais são os centros de pesquisa e os pesquisadores que mais se destacam no estudo de paradados coletados em questionários online?

Para responder a esta pergunta, foi verificada a filiação de cada autor, a fim de verificar qual é a maior concentração de centros de pesquisa onde ocorreram os estudos. Os resultados estão dispostos a seguir: Estados Unidos (13), Alemanha (7), Espanha (6), Reino Unido (3), Áustria (1), Bélgica (1), Eslovênia (1), Finlândia (1), Holanda (1), Itália (1), Jordânia (1), Nova Zelândia (1), Rússia (1) e Ucrânia (1). Estes países desenvolveram pesquisas abordando o emprego de paradados visando a garantia da qualidade da coleta de dados ou a experiência do usuário durante preenchimento de questionários online.

Liderando o ranking, a Universidade de Michigan (nos Estados Unidos), foi representada com oito estudos; a Espanha revelou a Universidade Pompeu Fabra, com sete estudos mapeados; na Alemanha, a Universidade de Göttingen e a Universidade de Mannheim sobressaíram na lista com cinco publicações para cada uma.

Quanto aos pesquisadores com maior destaque, são apresentados os autores que possuem mais de uma publicação neste mapeamento: Mick P. Couper (7), Melanie Revilla (6), Jan Karem Höhne (5), Stephan Schlosser (5), Carlos Ochoa (3), Tobias Gummer (3), Frederick Conrad (2), Gregg J. Peterson (2), Joss Rossmann (2), Vasja Vehovar (2).

Pergunta 2 - Como os estudos de paradados vêm se desenvolvendo ao longo dos anos?

Aqui, buscou-se o ano de publicação dos estudos nas bases consultadas. O resultado é apresentado a seguir: 2000 (1), 2003 (1), 2007 (1), 2008 (1), 2009 (1), 2010 (1), 2012 (1), 2013 (1), 2014 (1), 2015 (1), 2016 (4), 2017 (7), 2018 (4), 2019 (1), 2020 (10).

A partir de 2016, observou-se um aumento de publicações que atingiram o seu ponto mais alto em 2020, onde percebeu-se que o tema vem ganhando destaque a cada ano.

Entre os anos 2000 e 2003, apresentaram-se estudos iniciais sobre o tema. Os autores indicam possibilidades de uso dos paradados para avaliação da usabilidade do instrumento online de coleta 
e o registro de tempos de conclusão, a fim de ajudar a descrever como os informantes "construíram" suas respostas.

Entre 2007 e 2016, os estudos concentraram-se em avaliar o design visual do questionário, a fim de verificar os melhores formatos de perguntas e oferecer usabilidade para responder a pesquisa. Foram apresentados estudos comparativos entre elementos de interface, como: botões de opção (radio buttons) e menus suspensos (drop-down menus); escalas lineares (tipo Likert), caixas de seleção (check boxes) e listas; botões de opção, controles deslizantes (sliders) e escalas lineares; e discussões sobre a complexidade visual de grades (grids).

A partir de 2014, os pesquisadores começaram a se preocupar com o aumento do uso dos dispositivos móveis nas pesquisas, a fim de analisar se eram viáveis para questionários auto administrados na Web. Os pesquisadores começaram a pensar nas características dos equipamentos (como telas reduzidas e teclados virtuais) e no desafio de desenvolver questionários responsivos, otimizados com um design visual próprio para uso em dispositivos móveis.

Com relação aos usuários (informantes), os estudos refletiram que, desde 2015, existe a preocupação de estudar seus comportamentos enquanto preenchem os questionários. Dentre os estudos, podem-se citar a discussão sobre o tempo médio e o tempo ideal que os informantes precisam para responder a uma pergunta ou o questionário inteiro e os fatores que influenciam esta duração de acordo com o público-alvo; o rastreamento da navegação do usuário, através de suas ações com o mouse e o teclado; e a identificação de suas dificuldades para responder às perguntas, através de padrões de movimentos do mouse.

Os artigos mais recentes exploram descobertas da ciência, como examinar o comportamento do informante através da neurociência cognitiva. Os avanços da tecnologia também foram inseridos nos estudos, tais como: usar eye-tracking para identificar perguntas complexas, coletar os dados dos sensores dos smartphones para auxiliar nos estudos de qualidade das pesquisas; e permitir que o informante responda oralmente ao questionário na Web.

Pergunta 3 - Quais as bases acadêmicas e os veículos de divulgação científica que mais apresentaram o tema?

As buscas nas bases eletrônicas forneceram um panorama evolutivo da história e do desenvolvimento do conceito de paradados em um período de vinte anos de publicações, datadas entre 2000 e 2020.

Levantando as bases que mais publicaram sobre o tema, obteve-se o seguinte resultado: Scopus (16), ACM (10), SAGE (6), Springer Link (3), ScienceDirect (1). Quanto ao número de publicações ao longo dos anos, percebeu-se uma irregularidade nas quantidades das produções até 2012. A partir de 2013, as publicações anuais tornaram-se constantes e todas as bases citadas contribuíram com pelo menos um estudo acadêmico até 2020.

Na base $S A G E$, por se tratar de uma base dedicada às ciências sociais, metade de suas publicações referem-se a estudos comportamentais do informante ao preencher um questionário 
online. Por outro lado, os trabalhos publicados na $A C M$ em quase sua totalidade proporcionaram discussões sobre a interação do informante com a interface gráfica do questionário e a interação do informante com o dispositivo usado para responder o questionário.

Os jornais científicos periódicos foram os veículos de divulgação que mais publicaram os trabalhos. O Social Science Computer Review destacou-se com metade dos estudos deste mapeamento (18), seguido de: Computers in Human Behavior (3), Field Methods (2), International Journal of Market Research (2), Monitoring of Public Opinion: Economic and Social Changes (2), Sociological Methods \& Research (2), Journal of Emerging Technologies in Web Intelligence (1), Journal of Statistical Software (1), Metodoloski Zvezki: Advances in Methodology and Statistics (1), Quality \& Quantity: International Journal of Methodology (1) e Telematics and Informatics (1). Além dos periódicos citados, a conferência Human-Computer Interaction International, (que divulga novidades da área de interação humano-computador) fez parte do mapeamento, com dois estudos apresentados.

Pergunta 4 - Quais são as contribuições científicas dos estudos para o usuário?

As produções apresentam, em sua maioria, estudos comparativos, avaliações e recomendações, sendo intercaladas com outras contribuições. São elas: avaliações e estudos comparativos (7); avaliações, estudos comparativos e recomendações (7); estudos comparativos (7); avaliações e recomendações (4); recomendações (3); revisão da literatura e recomendações (3); aplicações (2); avaliações, estudos comparativos, frameworks ${ }^{1}$ e métodos (1); frameworks (1); frameworks, métodos, modelos e recomendações (1).

As recomendações costumam se referir a elementos de interface mais adequados para registrar as respostas; boas práticas de abordagem ao informante, de acordo com seu perfil demográfico; e possibilidades de aplicação, limitações, requisitos legais e éticos do uso dos paradados.

As revisões da literatura englobam: visão geral de métodos e abordagens que já foram usadas para avaliar a usabilidade de instrumentos de coleta; referencial teórico, descrevendo os principais erros de pesquisa e citando exemplos de paradados que podem minimizá-los; e revisão da literatura fornecendo uma lista de paradados que podem ser coletados em cada fase da pesquisa na Web.

As aplicações se referiram à apresentação de scripts para coletar as ações dos informantes, como abrir hiperlinks, interagir com os elementos da interface, inserir respostas, entre outros

Foram apresentadas também propostas de frameworks para rastrear e coletar as interações do usuário ao preencher um formulário na Web. Foi apresentado um framework teórico, baseado em teorias da neurociência cognitiva, a fim de compreender e aplicar a interação humana em uma interface gráfica de pesquisa online.

\footnotetext{
${ }^{1}$ Frameworks são modelos genéricos com diversas funcionalidades, que facilitam o desenvolvimento de uma aplicação.
} 
Os métodos listados incluíram a criação de uma forma para mensuração da satisfação das respostas em pesquisas na Web.

\section{Discussão sobre alguns resultados desta pesquisa}

Os estudos relacionados neste mapeamento exploraram os paradados para entender, explicar ou prever erros de preenchimento de questionários online, visando ao aprimoramento da experiência do usuário e à garantia da qualidade da pesquisa. Portanto, foi possível refletir sobre cada contribuição, para responder à questão primária do mapeamento: Como os paradados auxiliaram os pesquisadores nas análises de qualidade de questionários online?

Pretendeu-se ter uma visão geral dos paradados que foram adotados como ferramentas de investigação em pesquisas anteriores e refletir sobre oportunidades de aplicações futuras. Para isso, citações de uso de paradados nos estudos foram catalogadas e dispostas em ordem decrescente de aplicação: tempo de conclusão da pesquisa (23), informações sobre o dispositivo (19), tempo de resposta ao item (17), movimentos com o mouse (14), interrupção ou abandono do questionário (13), informações sobre o navegador (8), pressionamento de teclas ou movimentos de rolagem na tela (8), contato com o informante (7), alteração de respostas (6), dados ausentes (4), e informações sobre a conexão (3). A seguir, são apresentadas algumas discussões sobre os resultados.

Tempo de conclusão - Notou-se que o registro de tempo de conclusão do questionário foi o tipo de paradado mais utilizado, coletado em $64 \%$ dos trabalhos. Este paradado foi largamente observado em estudos comparativos de taxas de conclusão em diferentes dispositivos (computadores pessoais e dispositivos móveis). Os pesquisadores foram unânimes em afirmar que as pesquisas online concluídas em dispositivos móveis demoram mais do que as concluídas em um computador desktop. Este paradado ajuda a criar indicadores de duração média de preenchimento de questionários, o que pode ajudar a monitorar pesquisas que estão fora desta média.

Informações sobre o dispositivo, o navegador e a conexão à Internet - As informações sobre as características dos dispositivos despontam no segundo lugar da lista, coletadas em 53\% dos artigos. Tais paradados são úteis para conhecer qual dispositivo os informantes utilizam para responder às pesquisas e avaliar se o equipamento compromete a qualidade dos dados. Além das características dos dispositivos, outros dois detalhes técnicos foram coletados, a fim de permitir que os pesquisadores entendam o processo de navegação do usuário: informações sobre o navegador (22\%) e sobre a conexão à Internet (8\%).

Tempo de resposta de cada questão - Somando 47\% dos resultados, o registro dos tempos de resposta de cada questão aparece em terceiro lugar. Muito usados em conjunto com o tempo total de conclusão, este paradado foi coletado em avaliações sobre o efeito dos componentes da interface no formato da opção de resposta. Além disso, para os estudos de experiência do usuário, um tempo excessivo para responder pode ser sinal de problemas com o entendimento da pergunta. 
Movimentos com o mouse e pressionamento de teclas - Movimentos com o mouse e pressionamento de teclas (ou toques para a rolagem na tela) somaram 39\% e $22 \%$, respectivamente. Esses paradados foram coletados nos estudos para registrar ações significativas do usuário durante sua navegação em questionários online. Esses paradados ajudam a criar uma trilha de auditoria para detectar o comportamento de um usuário que pode estar confuso ou buscando orientação.

Interrupção ou abandono da pesquisa - As interrupções e abandonos de preenchimento dos questionários foram investigadas com o auxílio dos paradados em 36\% dos trabalhos, que concluíram que as pesquisas online tendem a ter altas taxas de interrupção e de abandono quando respondidas em dispositivos móveis. Segundo os estudiosos, as taxas de abandono são indícios de problemas; portanto, as análises visavam identificar o motivo pelo qual os informantes abandonaram o questionário. Tais paradados podem expor problemas técnicos, desinteresse pela pesquisa, falta de familiaridade do informante com a tecnologia ou problemas no design das questões.

Contato com o informante - Os paradados auxiliaram as análises quanto à abordagem ao informante em 19\% dos trabalhos. Esses estudos são relevantes para tentar mitigar a não resposta da unidade de pesquisa, o que revela a taxa indicadora de qualidade nas pesquisas: quanto menor a taxa de não-respostas (missing data), maior é a qualidade.

O contato inicial com o informante é fundamental. Em uma entrevista presencial, o entrevistador é treinado nos conceitos de pesquisa e em técnicas de acesso ao informante - a fim de criar um vínculo de confiança - para que este aceite responder a perguntas de uma pesquisa extensa e sensível, como o censo demográfico (TAVARES, 2011). Diferentemente da entrevista presencial, em uma pesquisa online o informante normalmente recebe um convite através de um e-mail, contendo informações para fazer seu login em um website. Reconhecendo a importância desta fase (chamada de "recrutamento"), diversos estudos dedicaram-se a aprimorar os textos dos e-mails de boas-vindas e torná-los personalizados para cada informante, a fim de buscar melhorar esse diálogo inicial. Foram empregados paradados para compreender o comportamento do informante e tentar minimizar possíveis recusas: o tempo de reação à pesquisa, o tempo para começar a responder a pesquisa, o número de tentativas para recrutar o informante, o número de lembretes ao informante, o número de visitas presenciais etc.

Alteração de respostas - Segundo os autores pesquisados, as mudanças de resposta por parte do informante também podem representar um indicador de problemas. A frequência com que respostas são alteradas foi observada em 17\% dos papers, e este indicador pode apontar eventuais erros a respeito do design visual das questões, assim como permite detectar problemas na redação de perguntas, sobre as quais os informantes podem estar apresentando dificuldades de compreensão.

Ausência de dados - À medida que a Internet vai sendo utilizada como meio para a coleta de dados nos censos demográficos, a compreensão da não resposta é cada vez mais importante. A não resposta a uma questão específica, analisada em 11\% dos trabalhos, ocorre quando a resposta 
é evasiva (por exemplo, "não sei" ou "prefiro não dizer") ou quando o informante decide ignorar a questão, direcionando-se para a questão subsequente. Como consequência, um questionário incompleto é enviado e resultados importantes podem ficar comprometidos.

Esses paradados podem contribuir para compreender os fatores que levam à não resposta como: a falta de familiaridade do informante ou o desinteresse sobre o tema; a não-compreensão do significado de uma pergunta; ou uma quantidade excessiva de opções de resposta que pode confundir os informantes, desencorajando-os. O design de telas inadequado também pode contribuir para a não resposta: excesso de elementos visuais, cores, sombreamento, fontes pouco legíveis, desorganização nos elementos, ou grids com excesso de colunas podem provocar reações negativas e até desestimular o informante.

\section{Conclusões}

Os censos demográficos nacionais exigem muito esforço de planejamento e realização. A forma tradicional de coleta de dados (visitas presenciais dos recenseadores aos domicílios) exige orçamentos elevados e enfrenta resistência das pessoas em receber recenseadores, o que torna os ciclos censitários cada vez mais difíceis de serem realizados. Por fim, a pandemia da Covid-19 reforçou a necessidade de repensar as coletas presenciais.

Tais problemas têm levado ao surgimento de propostas de coletas eletrônicas sem o auxílio de recenseadores, sendo que o Brasil tem realizado testes de coleta de dados pela Internet para o seu censo demográfico. No entanto, a migração do questionário do censo para a Web não é um processo simples.

$\mathrm{Na}$ abordagem online, o cidadão terá que responder ao questionário sem o apoio do recenseador. Há pontos críticos nesta transição, como: o nível de aceitação (pelos cidadãos) da opção Internet; a qualidade das informações; o tamanho e a navegabilidade do questionário; e a falta de entendimento das questões por parte do cidadão.

Destaca-se a importância da aplicação de princípios de UX na concepção de questionários autoadministrados pois, não havendo recenseadores, cabe ao sistema oferecer o suporte ao cidadão. Problemas no design do questionário e a não observação de princípios de usabilidade podem causar interpretações errôneas e prejudicar a qualidade dos dados.

Para monitorar o processo das pesquisas, dados auxiliares vêm sendo gradualmente implementados - os chamados paradados. Estes podem ser empregados como indicadores de desempenho das pesquisas ou para identificar fontes de erros em questionários, entre outros.

Neste contexto, este trabalho teve como proposta o mapeamento do estado da arte sobre paradados, a fim de verificar como podem apoiar avaliações de experiência do usuário em questionários online. Foram realizadas buscas em oito bases, retornando 906 artigos. Após a seleção, foram considerados trinta e seis estudos científicos para a análise de conteúdo. Pôde-se evidenciar o potencial dos paradados para as avaliações de UX, as bases acadêmicas com maior 
oferta de estudos, os centros de pesquisa e os pesquisadores com destaque no campo, a linha do tempo das publicações e as contribuições específicas de cada estudo.

Foi observado que a Scopus foi a base com maior produção neste mapeamento, seguida da ACM, SAGE, SpringerLink e ScienceDirect. O Social Science Computer Review destacou-se como o veículo de divulgação com mais publicações, contendo metade dos estudos selecionados. Os países com maior concentração de estudos foram os Estados Unidos, Alemanha, Espanha e Reino Unido, com mais de um centro de estudo em destaque. Os pesquisadores Mick P. Couper, Melanie Revilla, Jan Karem Höhne e Stephan Schlosser sobressaíram na lista, com trabalhos realizados em conjunto.

Percebemos que o tema vem ganhando destaque a cada ano, a partir de 2016. Os primeiros estudos foram básicos, sugerindo possibilidades de uso dos paradados. Depois, passaram a aplicar paradados para avaliar o design visual do questionário, para estudos de viabilidade de pesquisas em dispositivos móveis e para estudar o comportamento dos usuários enquanto preenchem o questionário. Os estudos recentes aplicaram descobertas da neurociência e os avanços da tecnologia para melhorar a interação com o informante.

O mapeamento forneceu também a visão sobre a produção científica dos estudos, que ofereceram avaliações, estudos comparativos, recomendações, revisão da literatura, aplicações, frameworks, métodos ou modelos como ferramentas para o diagnóstico de problemas relativos ao projeto da interface dos questionários.

Os paradados mostraram que o tempo de conclusão da pesquisa, informações sobre o dispositivo e o tempo de resposta de cada questão foram largamente observados pelos pesquisadores. Outros paradados, como movimentos com o mouse e interrupções no questionário, foram usados em escala menor, mas fundamentais para estudar o comportamento do usuário. Por fim, destacaram-se os paradados mais simples, mas com grande eficácia, como registros de contato com o informante, alteração de respostas e a indicação de ausência de dados.

A diversidade de paradados que podem ser coletados é ilimitada, à medida que a tecnologia vai evoluindo. Contudo, apesar da facilidade de coleta com baixo custo, não se deve subestimar o seu custo de gestão e análise. Além disso, existem questões sobre privacidade e ética ainda não discutidas. Os paradados não podem substituir outras formas de pré-teste dos questionários, mas podem ser uma boa ferramenta a ser empregada na avaliação de usabilidade, para uma melhor experiência do usuário durante o preenchimento do questionário online.

Espera-se que esta pesquisa gere algumas contribuições relevantes ao processo censitário brasileiro. Sob a perspectiva econômica, busca-se oferecer instrumentos eficazes de coleta de dados, na expectativa de reduzir o trabalho de campo, economizar no orçamento do censo e utilizar recursos da sociedade com maior eficiência. Sob a perspectiva da cidadania, a intenção é que os cidadãos tenham a liberdade de prestar suas informações na Internet, mantendo o direito de proteger a sua privacidade, sem deixar de cumprir o seu dever para com a coletividade. Sob a perspectiva acadêmica, pretende-se destacar a importância da investigação através de estudos remotos de interação com o usuário em ambientes reais, principalmente em tempos de pandemia. 
O presente mapeamento é parte de pesquisa de doutoramento que propõe o uso de paradados em conjunto com avaliações de usabilidade para questionários online, aplicados a censos demográficos, com a finalidade de identificar inconsistências e promover a coleta de dados eficiente. Desenvolvimentos posteriores deste trabalho envolverão detalhar estudos de caso, a partir do emprego de paradados, assim como empreender testes remotos de usabilidade.

Nota

O conteúdo do artigo é de inteira responsabilidade dos autores, não refletindo, necessariamente, a opinião do Instituto Brasileiro de Geografia e Estatística (IBGE).

\section{Referências}

AGNER, Luiz. Ergodesign e Arquitetura de Informação: Trabalhando com o Usuário. Rio de Janeiro: Editora Senac Rio, 2018. 240 p. $4^{\text {a }}$ edição.

CCSA. Committee for the Coordination of Statistical Activities. How COVID-19 is changing the world: a statistical perspective. 15 mai. 2020.

COUPER, Mick. Birth and Diffusion of the Concept of Paradata. Advences in Social Research \& Japanese Association for Social Research, 2017.

DURRANT, Gabriele; KREUTER, Frauke. Editorial: The Use of Paradata in Social Survey Research. Journal of the Royal Statistical Society. Series A (Statistics in Society), vol. 176, no. 1,2013 , pp. 1-3.

IBGE. Instituto Brasileiro de Geografia e Estatística. Censo Demográfico 2020 - $1^{\circ}$ Teste de Coleta pela Internet. IBGE. Agência de Notícias. 16 abr. 2018.

. Números mostram o valor do Censo para o avanço de políticas públicas no país. Agência IBGE. Revista Retratos. 17 jan. 2019.

Censo é adiado para 2021; coleta presencial de pesquisas é suspensa. IBGE. Agência de notícias. 17 mar. 2020.

INE. Instituto Nacional de Estatística. Estudo de viabilidade para os Censos 2021. Avaliação dos modelos censitários utilizados noutros países e a sua adequabilidade a Portugal. 30 de junho de 2014. Lisboa, Portugal.

KACZMIREK, Lars. Human-Survey Interaction Usability and Nonresponse in Online Surveys. 2008.

KITCHENHAM, Barbara; CHARTERS, Stuart. Guidelines for performing Systematic Literature Reviews in Software Engineering, 2007.

LEAL FERREIRA, Simone Bacellar; NUNES, Ricardo. 2008. e-Usabilidade, Rio de Janeiro, LTC.

NORDHOLT, Eric Schulte. The usability of administrative data for register-based censuses. Statistical Journal of the IAOS. IOS Press, 2018. doi:10.3233/SJI-180425.

PARSIFAL. Parsifal. Perform Systematic Literature Reviews.

PASC. Public Administration Select Committee. Options for the future of the census. UK Parliament. 25 February 2014. Reino Unido. 
TAVARES, Patricia Zamprogno. Estudo de usabilidade para PDAs utilizados em coleta de dados nas entrevistas pessoais para pesquisas domiciliares. Dissertação (Mestrado em Informática). Universidade Federal do Estado do Rio de Janeiro, Rio de Janeiro, 2011.

UNECE. United Nations Economic Commission for Europe. Conference of European Statisticians Recommendations for the 2020 Censuses of Population and Housing. Prepared in cooperation with the Statistical Office of the European Communities (EUROSTAT). 2015. United Nations Publications, Gênova, Suíça.

UNSD. United Nations Statistics Division. Statistics - COVID-19 response. Department of Economic and Social Affairs. 2020.

\section{Sobre os autores}

\section{Patricia Zamprogno Tavares}

Doutoranda pelo Programa de Pós-Graduação em Informática (PPGI) da Universidade Federal do Estado do Rio de Janeiro (UniRio). É analista de sistemas do Instituto Brasileiro de Geografia e Estatística (IBGE). Tem interesse nos seguintes temas: usabilidade, questionários eletrônicos, paradados e interação com o usuário. É membro do Núcleo de Acessibilidade e Usabilidade (NAU), grupo de pesquisa com foco nos aspectos de usabilidade e acessibilidade.

ORCID 0000-0003-0487-0460

CV Lattes http://lattes.cnpq.br/0002760226044780

\section{Luiz Agner}

Com pós-doutorado em Estudos Culturais pelo PACC-UFRJ, integra o quadro de designers do Instituto Brasileiro de Geografia e Estatística (IBGE), onde já atuou em projetos de Educação à Distância, em projetos de marketing, e na avaliação de usabilidade de dispositivos móveis de coleta de dados. Professor de Comunicação Social das Faculdades Integradas Hélio Alonso (Facha), é autor do livro "Ergodesign e Arquitetura de Informação - Trabalhando com o Usuário" (quarta edição - Editora Senac).

ORCID 0000-0001-6101-1991

CV Lattes http://lattes.cnpq.br/2328427339458925

\section{Simone Bacellar Leal Ferreira}

Professora Associada dos cursos de Sistemas de Informação (doutorado, mestrado e graduação) do Departamento de Informática Aplicada da Universidade Federal do Estado do Rio de Janeiro (UniRio); fundadora e coordenadora do NAU - Núcleo de Acessibilidade e Usabilidade da UniRio. Áreas de atuação e interesse: Interação Humano-Computador (Usabilidade e Acessibilidade), Sistemas de Informação e Uso de Cores. Autora do livro e-Usabilidade, publicado e lançado em setembro de 2008.

ORCID 0000-0001-9031-0122

CV Lattes http://lattes.cnpq.br/0926018459123736 